\author{
SERIES 'CLINICAL PHYSIOLOGY IN RESPIRATORY INTENSIVE CARE' \\ Edited by A. Rossi and C. Roussos
}

\title{
Computed tomography in adult respiratory distress syndrome: what has it taught us?
}

\author{
P. Pelosi, S. Crotti, L. Brazzi, L. Gattinoni
}

Computed tomography in adult respiratory distress syndrome: what has it taught us? $P$. Pelosi, S. Crotti, L. Brazzi, L. Gattinoni. CERS Journals Ltd 1996.

ABSTRACT: Computed tomography (CT) has played an important role in improving our knowledge of the pathophysiology of the adult respiratory distress syndrome (ARDS), and in determining the morphological and functional relationships of different manoeuvres commonly used in the therapeutic management of this syndrome (changes in body position, application of positive end-expiratory pressure (PEEP) and mechanical ventilation).

During the early phase of the disease, the ARDS lung is characterized by a homogenous alteration of the vascular permeability. Thus, oedema accumulates evenly in all lung regions with a nongravitational distribution (homogenous lung). The increased lung weight, due to increased oedema, causes a collapse of the lung regions along the vertical axis, through the transmission of hydrostatic forces (compression atelectasis). Thus, the lesions appear mainly in the dependent lung regions (dishomogeneous lung). During inspiration, at plateau pressure, the pulmonary units reopen and, if the PEEP applied is adequate, they stay open during the following expiration. Adequate PEEP is equal to or higher than the hydrostatic forces compressing that unit. Prone position is another manoeuvre which allows previously collapsed lung regions to reopen and, conversely, compresses previously aerated regions, reversing the distribution of gravitational forces. During late ARDS, there is less compression atelectasis and the lung undergoes structural changes, due to the reduced amount of oedema. This is usually associated with $\mathrm{CO}_{2}$ retention and the development of emphysema-like lesions.

In conclusion, computed tomography is not only a research tool, but a useful technique which allows a better understanding of the progressive change in strategy needed to ventilate the adult respiratory distress syndrome lung at different stages of the disease.

Eur Respir J., 1996, 9, 1055-1062.
Istituto di Anestesia e Rianimazione, Università di Milano, Ospedale Maggiore IRCCS, Milano, Italy

Correspondence: P. Pelosi

Istituto di Anestesia e Rianimazione

Università di Milano

Ospedale Maggiore

via Francesco Sforza 35

20122 Milano

Italy

Keywords:

Adult respiratory distress syndrome computed tomography

positive end-expiratory pressure

prone position

mechanical ventilation

Received: December 151995

Accepted for publication January 301996
In the last $10 \mathrm{yrs}$, the use of computed tomography (CT) in the clinical evaluation of thoracic diseases has rapidly gained popularity and CT has become firmly established as an important research and diagnostic modality [1]. In fact, CT makes it possible to obtain cross-sectional imaging of the lung, so that virtually all intrathoracic pathological conditions can be evaluated. In particular, CT has played an important role in improving our knowledge of the pathophysiology of the adult respiratory distress syndrome (ARDS) and in determining the morphological and functional relationships of different manoeuvres commonly used in the therapeutic management of this syndrome [2].

ARDS refers to a generalized inflammation of the lung parenchyma, caused by different diseases. ARDS is characterized by diffuse pulmonary infiltrates, decreased respiratory compliance and severe hypoxaemia, barely responsive to the administration of high inspiratory oxygen concentrations [3]. Moreover, the pathological characteristics of ARDS may change during the course of the disease, from oedema to fibrosis and disruption of alveolar septa [4].
The purpose of this review is to briefly summarize the role of CT in defining the pathophysiology of the ARDS lung and the possible therapeutic, clinical implications of these findings. In particular, we will focus on: 1) the technical methodological aspects of CT; 2) the lung structure-function relationships in early ARDS; 3) the effects of different therapeutic manoeuvres, such as changes in body position, application of positive end-expiratory pressure (PEEP) and mechanical ventilation, on the lung structure; and 4) the alterations of lung function occurring with time.

\section{Technical aspects}

The quantitative approach to the CT scan relies on the analysis of "CT numbers", which substantially define the density (i.e. mass/volume) of each voxel composing the image $[5,6]$. The entire image is divided into several voxels (dimensions $1.5 \times 1.5 \times 9 \mathrm{~mm}$ ) characterized by a CT number that is expressed in Hounsfield units (HU), ranging from $+1,000 \mathrm{HU}$ (bone) to $-1,000 \mathrm{HU}$ (air), with 
the density of water $=0 \mathrm{HU}$. Thus, a voxel with $\mathrm{CT}$ equal to $-500 \mathrm{HU}$ can be considered as constituted by $50 \%$ gas and $50 \%$ tissue (tissue including lung structures, extravascular lung water, blood and fibrin).

Knowing the density and measuring the total lung volume either by the helium dilution technique [7] or directly by the CT scan image [8], it is possible to compute for the entire lung or a given lung region: 1) the amount of gas; 2) the amount of "tissue"; and 3) the gas/tissue ratio. The gas/tissue ratio is taken as a quantitative estimation of the degree of alveolar inflation. Moreover, knowing the lung density and the lung height (from sternum to vertebra, in supine position) at different lung levels, it is possible to estimate the superimposed hydrostatic pressure at that height (level), assuming that the transmission of the pressures throughout the lung parenchyma is similar to that in a liquid (pressure at a given height is equal to density $\times$ height $)$.

Taking the CT scan under different lung conditions, it is then possible to calculate the alveolar recruitment and the distribution of ventilation. Alveolar recruitment is defined as the amount of tissue without gas which regains inflation after application of different inflation or deflation pressures. The distribution of ventilation is, indeed, obtained as the difference in alveolar inflation between end-inspiration and end-expiration, in static conditions. Finally, the same measurements make it possible to investigate the modification in lung morphology with changes in body position, for instance, when the patient is turned from supine to prone. It is, thus, evident that CT scan analysis allows the computation of several variables, leading to a description "in vivo" of the lung pathology and structure-function relationships in ARDS.

\section{Lung-structure function relationships in early ARDS: homogenous or dishomogeneous lung?}

ARDS is characterized by pulmonary infiltrates, which were previously considered to be rather homogeneously distributed throughout the lung, as evidenced by conventional chest radiograph $[3,9]$. However, using CT technology, it has been shown that in the ARDS lung the localization of radiographic densities is primarily located in the dependent lung regions, i.e., the vertebral regions (lower) in supine position $[5,10,11]$. In contrast, the nondependent regions, i.e. the sternal regions (upper) in supine position, seem, at least to visual inspection, quite normal (fig. 1). These findings have challenged the commonly held opinion that ARDS is a generalized lung disease.

This configuration of dishomogeneous lung is similar and only quantitatively different from what has been reported in normal subjects after anesthesia and paralysis $[12,13]$. In fact, during anaesthesia, normal lungs appear rather dishomogeneous, since they are characterized by the presence of a variable amount of densities in the dependent part of the lung, occupying, on average $8-10 \%$ of the entire lung field. The appearance of these densities has been attributed to the development of "compression atelectasis", i.e., to the loss of gas in the most dependent part of the lung. Moreover, the degree of hypoxaemia, occurring after induction of anaesthesia,

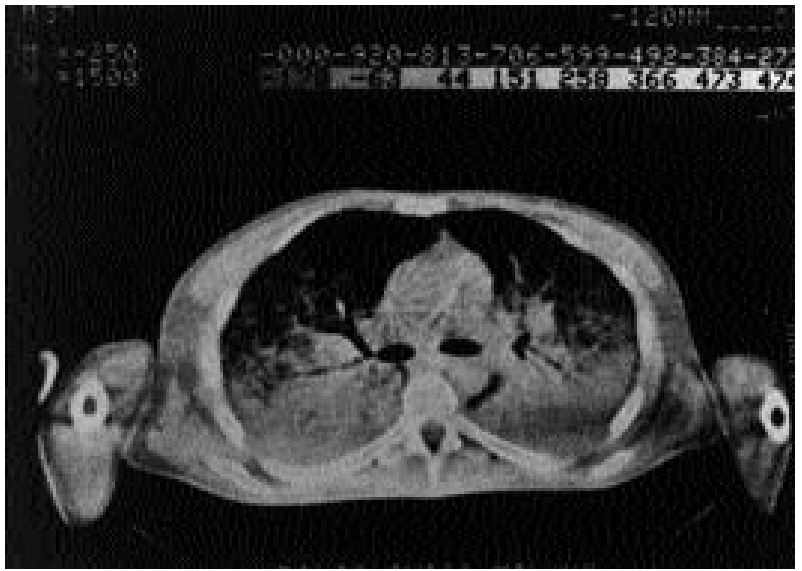

Fig. 1. - Typical example of adult respiratory distress syndrome (ARDS) as seen with computed tomography. The densities are primarily located in the dependent lung regions.

related well to the quantitative amount of these atelectatic regions [14].

In ARDS patients the densities may account for up to $70-80 \%$ of the lung field, depending on the severity of respiratory failure. The proportion of lung which can be ventilated can consequently be reduced to almost 20 $30 \%$ of a normal lung and, therefore, may have the dimensions of the lung of a baby. For these reasons, it has been called "baby lung" [15]. The dishomogeneity of the distribution of lung lesions with a sparing of the nondependent part of the lung, first led to modelling of the ARDS lung as a dishomogeneous lung, composed of three compartments: one substantially normal (healthy zone); one fully diseased without any possibility of recruitment (diseased zone); and, finally, one composed of collapsed alveoli potentially recruitable with increasing pressure (recruitable zone) [16]. The presence of the diseased and recruitable compartments may account for marked increase in CT density in ARDS lung compared to normal $[7,17]$. In fact, the overall CT density of ARDS lung is approximately three times greater compared with the lung of normal subjects $(-256 \pm 21 v s-654 \pm 8 \mathrm{HU}$, respectively), due to marked differences in the amount of tissue i.e. oedema $\left(31.6 \pm 1.7\right.$ vs $16.7 \pm 0.8 \mathrm{~mL} \cdot \mathrm{m}^{-2}$ body surface area, respectively) and gas volume $(11.5 \pm 1.2 \mathrm{vs}$ $32.2 \pm 1.8 \mathrm{~mL} \cdot \mathrm{m}^{-2}$, respectively). However, the overall volume of the thorax is not substantially different between ARDS and normal patients $(43.0 \pm 2.3$ and $49.0 \pm 2.5$ $\mathrm{mL} \cdot \mathrm{m}^{-2}$, respectively), suggesting that in ARDS the decrease in lung volume does not indicate a decrease in total thoracic volume, but a simple replacement of gas volume with tissue volume. This results in an increase in lung weight by 2-3 fold compared to normal [7].

The reduction in respiratory compliance, a typical finding in ARDS, was previously attributed to the severity of the disease or to intrinsic mechanical alterations of the lung tissues. Using CT, we demonstrated that, contrary to a commonly accepted notion, respiratory compliance was not related to the amount of "diseased" tissue but to the amount of residual inflated lung, indicating that the smaller the portion of lung open to gas, the lower was the compliance [16].

Whereas respiratory compliance was correlated with the normally inflated part of the lung, the gas exchange 
impairment was strongly related to the amount of noninflated tissue mass, i.e. to the extent of the disease [16]. This suggests that the shunt fraction, due to the perfusion of the noninflated tissue, is probably the main cause of severe hypoxaemia in ARDS patients. Moreover, it may be hypothesized that the dependent lung regions, where the majority of noninflated tissue is present, may be underperfused, as evidenced by the discrepancy between the shunt fraction, and the noninflated tissue fraction (i.e. $30 \%$ of shunt with $60 \%$ of noninflated tissue). The underperfusion of these regions could be due both to mechanical compression of the vessels and to a possible hypoxic pulmonary vasoconstriction [18].

Interestingly, a positive correlation was also found between pulmonary artery pressure and the excess tissue mass; about $1 \mathrm{mmHg}$ of pulmonary artery pressure was associated with a $14 \%$ increase of the original lung weight. Unfortunately, it was impossible to determine whether the excess tissue mass was increasing the pulmonary artery pressure or otherwise [7]. However, this finding suggests that careful control of pulmonary artery pressure is mandatory in this type of patient. Moreover, all therapeutic manoeuvres which might decrease pulmonary artery pressure, such as reduction in mean airway pressure or use of pulmonary vasodilators, should always be attempted in the clinical management of these patients.

Considering the complexity of the anatomical changes occurring in ARDS lung structure, regional CT analysis allowed the anatomical changes occurring in ARDS to be described more exactly. In fact, using the regional CT analysis of the lung (fig. 2), we greatly improved our knowledge of the pathophysiology of ARDS, partially modifying some of the previously well-established concepts. We found that, contrary to previous findings, all of the lung parenchyma was homogeneously affected by the disease, with no part of the lung being healthy, and oedema did not exhibit a gravity-dependent distribution along the vertical gradient, in supine position (fig. 3). Consequently, it did not accumulate preferentially in the dependent lung regions. This finding is in accordance with previous reports obtained in a number of animal experiments of respiratory failure using different measurement techniques [19-21] and in cardiogenic pulmonary oedema [22], where a nongravitational distribution of oedema has been described. The reasons why oedema does not distribute according to the gravity are substantially unknown; however, it appears that the primary mechanism leading to oedema, at least in ARDS, acts equally in each part of the lung and that oedema cannot

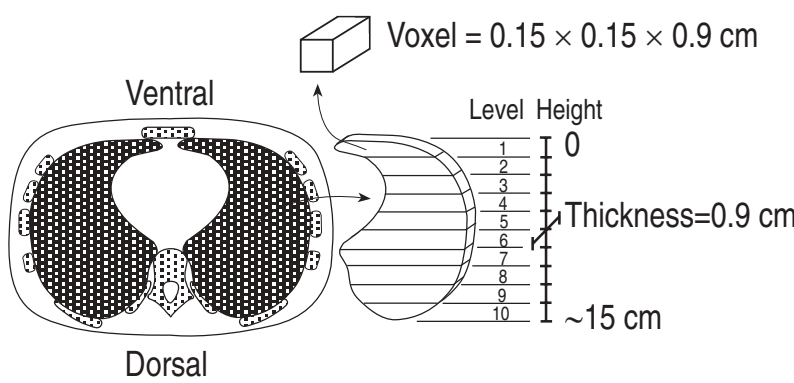

Fig. 2. - Procedure for regional image analysis: the computed tomography section of the lung is contoured and the total height is divided into 10 equal intervals defining lung levels (From [12], with permission).

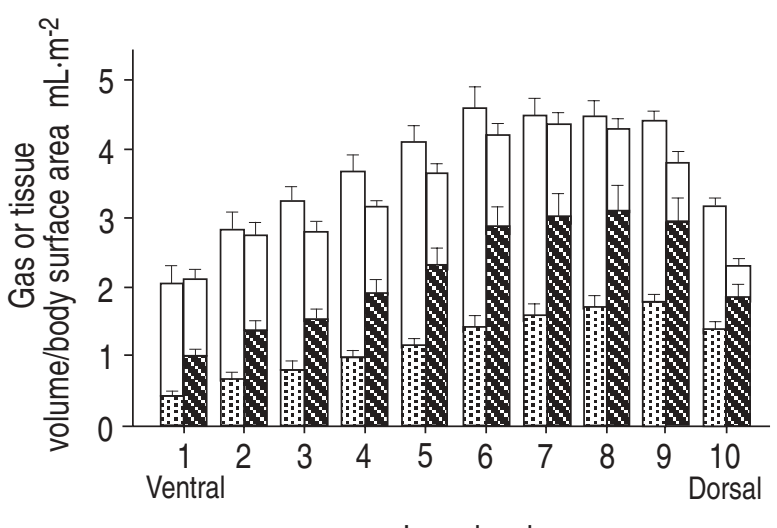

Lung level

Fig. 3. - Tissue and gas volumes in supine lung levels 1 (ventral) to 10 (dorsal) in normal subjects (first bar in each pair) and in patients with adult respiratory distress syndrome (ARDS) (second bar in each pair). The total volume of the levels was comparable between the two groups, whilst the gas volume was lower and tissue volume higher in each level in ARDS compared to normal. Data are presented as

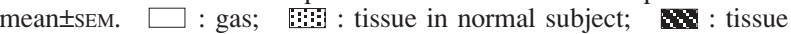
in patient with ARDS. (From [12], with permission).

move freely through the interstitial space. However, as mentioned before, the ARDS lung appears inhomogeneously affected and, from ventral to dorsal lung regions, the CT density increases and regional inflation decreases, suggesting a progressive deflation of gas content in the alveoli along the vertical gradient.

How may a homogeneous distribution of the disease coexist with the appearance of dishomogeneous distribution of densities, prevalently located in the dependent lung regions? This may occur because the oedematous ARDS lung is heavier than a normal one and it is placed in a gravitational field. In fact, it has been shown that the superimposed pressure overlying each lung level from nondependent to the dependent regions, is markedly increased compared to normal (fig. 4). Assuming that pressures are transmitted through the lung parenchyma as in a fluid (a fluid-like model) it may be hypothesized that the transpulmonary pressure $(P$ tp $)$ gradient is substantially increased in ARDS patients. It is well-recognized that the local $P$ tp is the main factor responsible for

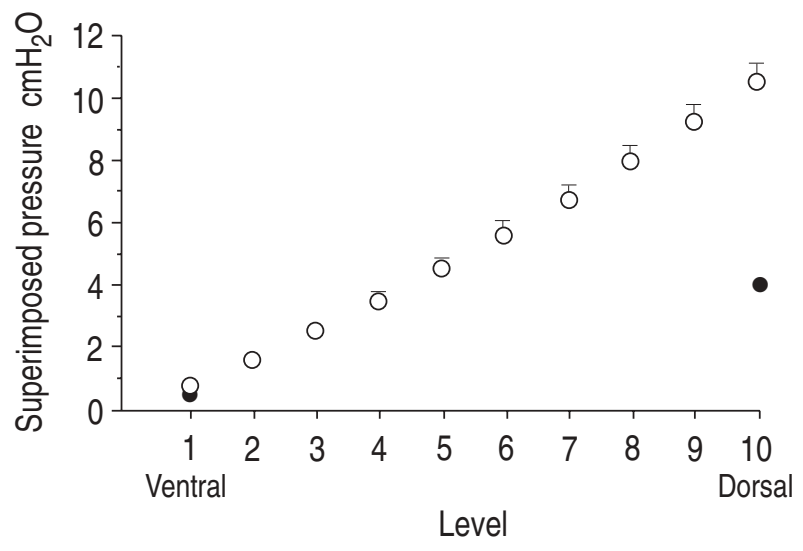

Fig. 4. - Superimposed pressure in patients with adult respiratory distress syndrome (ARDS) (O). Reference data for normal $(\bullet)$ are: superimposed pressure $0.5 \pm 0.1 \mathrm{cmH}_{2} \mathrm{O}$ at level 1 and $4 \pm 0.5 \mathrm{cmH}_{2} \mathrm{O}$ at level 10 [12]; gas/tissue ratio $6 \pm 0.3$ at level 1 and $2 \pm 0.2$ at level 10 [12]. Data are presented as mean \pm SEM. 
the regional inflation gradient, but the nature of the vertical $P$ tp gradient remains substantially controversial [23, 24]. It may be due to the lung weight, the shape and mechanical properties of the chest wall, and/or the shape and mechanical properties of the lung. However, at least in humans, the role of lung weight is considered prevalent [24]. As a consequence, whatever the role of the thoracic or lung shape, in ARDS the increased regional superimposed pressure is the major factor explaining the increased regional inflation gradients, since it causes lung deflation and collapse along the vertical gradient due to an increased weight [17].

These observations have led to some modification of the concept that the ARDS lung is characterized by a dishomogeneous disease (the three zone model of lung described previously). Even if the lung densities appear prevalent in the dependent lung regions (dishomogeneous lung disease), the oedema accumulates homogeneously throughout the lung parenchyma (homogeneous lung disease). Thus the nondependent lung regions, which appeared quite normal at a visual inspection, are in fact affected by the disease. We thus hypothesized that the increased weight, due to the presence of oedema and the resulting increased hydrostatic forces, cause a progressive squeezing of the gas along the ventral-dorsal axis, due to the gravitational field, resulting in the dependent lung being almost collapsed.

\section{Effects of changes in body position}

Prone positioning is a commonly accepted manoeuvre to improve oxygenation in ARDS patients $[25,26]$. However, the mechanisms leading to this improvement has not been yet completely clarified.

We found that a few minutes after body position change from the supine to prone, the densities moved from dorsal to ventral regions $[8,25,26]$. Thus, according to the model described above, the densities moved from the previously dependent to the new dependent regions of the lung due to a different application of gravitational forces. This rapid redistribution can hardly be explained by formation of new oedema in the dependent regions and reabsorption of the earlier oedema in the nondependent lung regions. In fact, reabsorption of oedema usually takes hours or even days to occur. Moreover, the changes in the CT densities are too great to be explained only by a shift of blood volume. Consequently, the most likely explanation is the redistribution of intrapulmonary gas, caused by the reverse of increased hydrostatic pressures (superimposed pressure). The hydrostatic forces in supine position collapse the dorsal lung (the most dependent one), whilst in prone position the most dependent lung, with higher hydrostatic forces, is the ventral one. Thus, the ventral lung regions collapse and the dorsal regions are recruited.

However, we were not able to find any relationship between redistribution in lung densities during positioning and modifications in oxygenation. Thus, it is possible that many factors may account for the gas exchange improvement with positioning. The most probable factor is a more favourable distribution of ventilation and perfusion, resulting in an improved ventilation/perfusion relationship [27].

\section{Physiological effects and mechanism of PEEP}

The response to PEEP in terms of respiratory mechanics, oxygenation and haemodynamics varies in ARDS patients [28, 29]. In fact, in some patients PEEP increase leads to a significant improvement of gas exchange, whilst in other patients it does not. Using the CT scan, the morphological basis of the PEEP response has been studied. The effects of PEEP on density are immediate (5-10 min) and no further clearing of lung densities has been observed for at least $1 \mathrm{~h}$ after application of a new PEEP level. Moreover, when there is a prevalence of recruitable tissue the PEEP response is beneficial, with an increase in oxygenation and less effect on haemodynamics [7, 30].

Again the regional analysis of the lung by CT scan has allowed the mechanism through which PEEP improves oxygenation in patients with ARDS to be better defined. PEEP may increase lung volume either through greater inflation of open pulmonary units or by recruiting collapsed pulmonary units.

To test the hypothesis that PEEP acts by counteracting the increased superimposed pressure at each lung level, and thus reducing the collapse of alveolar regions due to pulmonary shunt and hypoxaemia, we studied 10 sedated, paralysed ARDS patients at several PEEP levels (from 0 to $20 \mathrm{cmH}_{2} \mathrm{O}$ ) at end-expiration [31]. This made it possible to construct a gas/tissue ratio-pressure curve at each lung level. Three different types of curve were obtained: 1) a straight line, suggesting the presence of open pulmonary units which inflate according to their own compliance; 2) a biphasic curve, which presents an inflection point, suggesting the presence of collapsed units which are recruited only when a critical pressure is applied; 3) a nonsignificant gas/tissue ratio-pressure curve, indicating that the gas/tissue ratio does not increase with pressure at any point, suggesting the presence of consolidated pulmonary units. If our hypothesis is correct, we would expect that the inflection point at a given lung level should be equal to the pressure superimposed at that level. In fact, we found that the nondependent lung was characterized by straight line gas/tissue ratio-pressure curve (type 1), while the frequency of biphasic response (type 2) increased along the ventral-dorsal axis, as well as the inflection point pressure $(P$ flex). More importantly, we found an equivalence between the inflection point and the superimposed pressure (fig. 5).

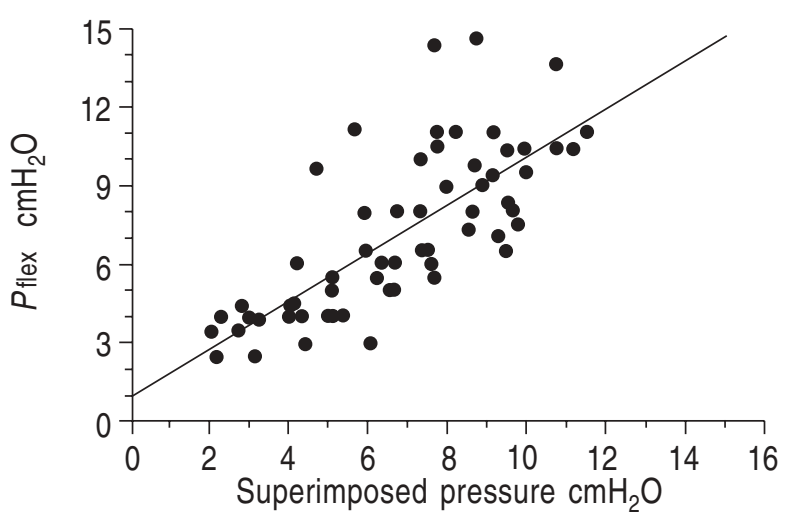

Fig. 5. - Relationship between inflation point pressure $(P$ flex $)$ as a function of superimposed pressure $(P \mathrm{sp})$, is described by the following equation: $P_{\text {flex }}=1.05+0.9 \times P_{\mathrm{sp}}(\mathrm{r}=0.75 ; \mathrm{p}<0.01)$. (From [31], with permission). 
All of this information combined indicates that the increased hydrostatic pressure along the vertical gradient (due to the increased oedema) causes compression of the underlying alveoli, allowing the formation of atelectasis in the dependent lung; PEEP acting as a counterforce, keeping open the previously collapsed pulmonary units and, thus, reducing the amount of nonaerated tissue. Obviously, PEEP and the prone position probably share the same mechanism of action, i.e. counteracting, in different ways, the superimposed pressure which causes compression atelectasis. In fact the higher the amount of collapsed (not consolidated) units, which may be recruited with PEEP, the higher is the redistribution of densities when changing position [19].

These new findings regarding the mechanism of action of PEEP may have different clinical implications. Firstly, applying PEEP is always a compromise, since the reopening of the most dependent lung regions is paralleled by a further increase in the alveolar inflation of the nondependent ones. Secondly, the maximal PEEP to be applied in the supine position to inflate the pulmonary units that underwent compression atelectasis should not exceed the ventral to dorsal height of the lung in centimetres. In fact, even given the hypothesis that the whole lung is completely filled with water, the maximal pressure to be applied to reopen the most dependent lung regions should be equivalent to the lung height, since the specific weight of the water is $1 \mathrm{~g} \cdot \mathrm{mL}^{-1}$.

\section{Effects of mechanical ventilation: distribution of ventilation and recruitment}

All of the considerations above refer to the ARDS lung as it presents at end-expiration, in static conditions. Recently, we examined the behaviour of regional distribution of gas volume and recruitment both at end-expiration and at end-inspiration at different levels of PEEP (from 0 to $20 \mathrm{cmH}_{2} \mathrm{O}$ ) in a group of eight sedated, paralysed ARDS patients during volume-controlled ventilation [32]. We found that ventilation at PEEP $0 \mathrm{cmH}_{2} \mathrm{O}$, in supine position, was distributed mainly in the upper lung, the ratio between the amount of ventilation in the upper and lower lung being approximately 2.5:1 with increased PEEP, the distribution of the inspired tidal volume became progressively more homogeneous, the ratio being approximately $1: 1$ at $20 \mathrm{cmH}_{2} \mathrm{O}$ of PEEP (fig. 6). It should be noted that measurements were obtained in static conditions, thus avoiding the possible effects of respiratory resistance. If the time constants of the different lung regions are similar, the dynamic distribution of ventilation would follow similar patterns to those described in static conditions. In fact, in ARDS airway resistances are related mainly to the lung volume [33]. As a consequence, we would expect resistances to be lower in the more inflated part of the lung (upper regions) and higher in the dependent lung. Moreover, the nonohmic resistances, called "tissue resistance" [34, 35] should also be greater in the dependent lung when continuing collapse and decollapse of the lung tissue occurs. Consequently, in our opinion, at least during mechanical ventilation, the results that we obtained in static conditions may be extrapolated to dynamic conditions.

This implies that modifications to regional compliance occurred with PEEP, in particular a decrease of

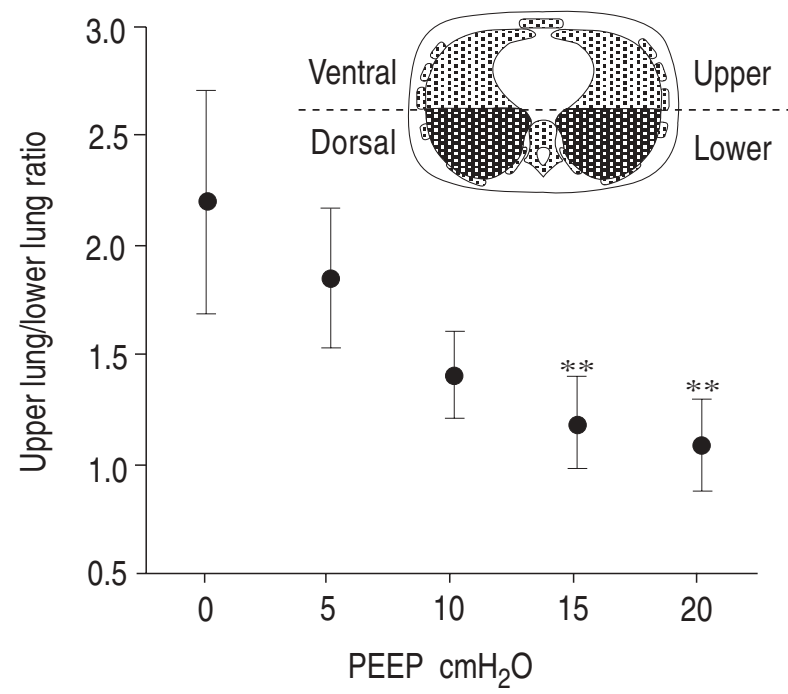

Fig. 6. - Distribution of ventilation, expressed as the ratio between the tidal volume delivered in the upper (levels 1-5) and lower (levels 6-10) lung compartments. Data are presented as mean \pm SEM. **. $\mathrm{p}<0.01$, compared to $0 \mathrm{cmH}_{2} \mathrm{O}$ PEEP. (From [32], with permission).

compliance in the upper lung, no change in the middle lung and an increase in the lower levels (fig. 7). Compliance may depend on several factors, among them the intrinsic elasticity of the pulmonary units (usually estimated as specific compliance) and the number of alveolar units that can be ventilated. Since in ARDS, the specific compliance is reported to be quite normal in each lung region $[31,32]$, whilst the number of open pulmonary units at functional residual capacity (FRC) decreases from nondependent to dependent lung regions, modifications in ventilation distribution are probably due to changes in regional compliance.

Regional analysis of recruitment provided a possible explanation to this phenomenon. In fact, in the upper levels where the superimposed pressure is low and atelectatic regions are few, recruitment is negligible and overdistension is present, whatever inspiratory pressure is applied. In contrast, in the middle and lowest levels, where the superimposed pressure is higher thus possibly causing some atelectasis, application of PEEP may cause recruitment, increasing regional compliance. We also showed that during mechanical ventilation and tidal volume delivery a considerable part of the lung continuously collapses and reinflates, especially at low levels of PEEP, when a greater part of the lung is collapsed at end-expiration. This phenomenon obviously occurs mainly in the most dependent regions, where compression atelectasis is prevalent. With PEEP, the reinflation-collapsing tissue decreased, since the amount of collapsed tissue at end-expiration decreased.

However, this study was performed maintaining a constant tidal volume, resulting in an increase in peak pressure when PEEP was changed. Thus, it was difficult to determine the relative importance of the pressure level reached at end-inspiration (peak pressure) or at end-expiration (PEEP) to changes in ventilation and recruitment. Consequently, further investigations are warranted to better define the interactions between peak pressure and PEEP in determining the distribution of ventilation and recruitment, thus providing new insights into the possible mechanisms causing lung damage. 
a)

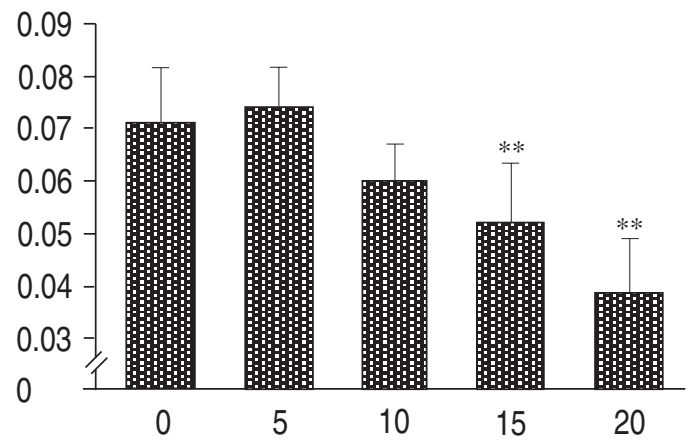

b)

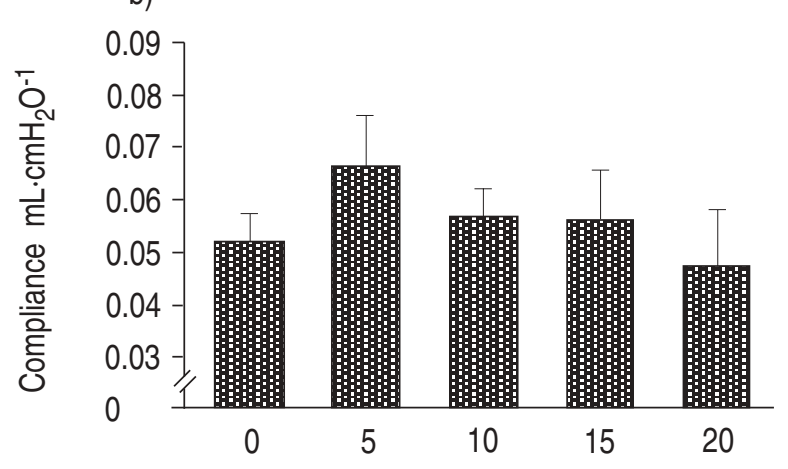

c)

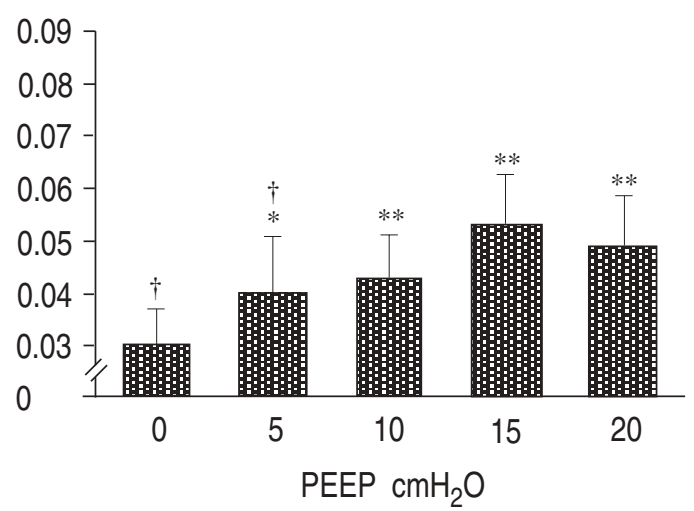

Fig. 7. - Compliance distributed in each of the: a) upper (1-3); b) middle (4-7); and c) lower (8-10) lung levels, at different PEEP. Data are expressed as mean \pm SEM. a) The compliance significantly decreased with PEEP $(\mathrm{p}<0.01)$. b) The compliance did not change with PEEP NS). c) The compliance significantly increased with PEEP $(p<0.01)$. Statistical significance for individual comparisons: *: $\mathrm{p}<0.05$, compared to $0 \mathrm{cmH}_{2} \mathrm{O}$. **: $\mathrm{p}<0.01$, compared to $0 \mathrm{cmH}_{2} \mathrm{O}$. $\dagger$ :Vertical comparisons; at 0 and $5 \mathrm{cmH}_{2} \mathrm{O}$ PEEP, the compliance decreased significantly $(\mathrm{p}<0.01)$ from upper to lower lung levels. No significant differences were found at 10, 15 and $20 \mathrm{cmH}_{2} \mathrm{O}$ PEEP. PEEP: positive end-expiratory pressure.

Finally, our data suggest that great attention should be taken to the precise timing of the performance of CT of the lung; in the same patient, different CT images may appear if taken at end-expiration or at end-inspiration, i.e. at different pressure levels. This may lead the physician to a mistaken interpretation of the CT scan image. Taken together, the information obtained with CT scan may be particularly useful in identifying and titrating the optimal ventilatory setting in this type of patient, adopting the maximal PEEP level to recruit atelectatic lung regions, avoiding excessive overdistension during tidal volume ventilation.

\section{Lung structure and function in ARDS evolution with time}

Data regarding the effects of duration of mechanical ventilation on lung structure of ARDS patients are astonishingly scanty. The application of CT in ARDS made it possible to detect in vivo the changes in lung morphology occurring during prolonged use of mechanical ventilation [36]. The most important finding is the presence of cysts and emphysema-like lesions prevalent in the dependent lung regions with the stage of disease andduration of mechanical ventilation (fig. 8). In contrast, the overall lung density decreases and becomes spread throughout all of the lung parenchyma instead of being prevalent in the most dependent part of the lung. In late ARDS, the emphysema-like lesions are associated with an increased incidence of pneumothorax as well as an increase in dead space and arterial carbon dioxide tension $\left(\mathrm{Pa}, \mathrm{CO}_{2}\right)$. Moreover, in late ARDS the development of lung fibrosis reduces the transmission of hydrostatic forces with a consequent decrease in the occurrence of atelectasis in the dependent lung. It is, thus, evident that the lung structure and function markedly change with the duration of mechanical ventilation. Consequently, it appears that the PEEP requirement in late ARDS is decreased, as compression atelectases are no longer present in the late stages of the disease.

\section{Conclusion}

By use of CT in ARDS, previously established concepts concerning the pathophysiology and the mechanism of action of different therapeutic manoeuvres, such as PEEP, volume-controlled ventilation and changes in body position have been challenged. In fact, ARDS is a homogeneous disease, characterized by a uniformly increased lung oedema and lung weight. However, it presents as a dishomogeneous disease because of the

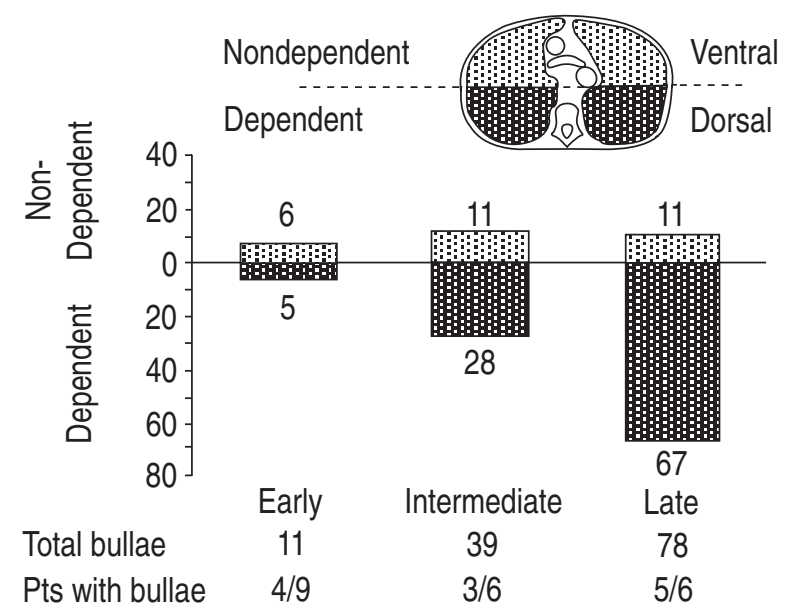

Fig. 8. - Prevalence of emphysema-like lesions in the nondependent lung and the dependent lung in early, intermediate and late adult respiratory distress syndrome (ARDS). The number of bullae was significantly higher in the dependent lung in intermediate and late stage ARDS compared with early ARDS $(\mathrm{p}<0.01)$. Moreover, the number of patients (Pts) with bullae was significantly higher in intermediate and late ARDS compared to early ARDS. (From [36], with permission). 
effect of the gravitational field on this heavy lung causing the formation of atelectases, especially in the dependent part of the lung. Different ventilatory strategies, such as the use of changes in body position, PEEP and different tidal volumes, may strongly influence the structural modifications of the lung. Some of these are, indeed, beneficial, when causing alveolar recruitment, whilst others are extremely disadvantageous, when causing alveolar overdistension. Thus, CT scan may be useful in titrating the "optimal" ventilatory strategy. Finally, the application of CT in ARDS has made it possible to better define the structural modifications in the lung caused by the duration of mechanical ventilation, providing evidence that different ventilatory strategies should be performed according to the stage of the disease.

All of this information has not only improved our pathophysiological knowledge concerning the development of adult respiratory distress syndrome, but may have interesting consequences for the therapeutic management. However, further studies should be performed to better define the role of computed tomography in the routine clinical management of adult respiratory distress syndrome patients.

\section{References}

1. Naidich DP, Webb WR, Muller NL. Thoracic computed tomography: current concepts. In: Potchen EJ, Grainger RG, Greene R, eds. Pulmonary Radiology. Philadelphia, Saunders Co., 1993; pp. 386-404.

2. Gattinoni L, Pelosi P, Brazzi L. Computed tomography in adult respiratory distress syndrome. Respir Ther 1995; 4: 79-83.

3. Ashbaugh DG, Bigelow DB, Petty TL, et al. Acute respiratory distress in adults. Lancet 1967; ii: $319-323$.

4. Teplitz C. The core pathobiology and integrated medical science of acute respiratory insufficiency. Surg Clin North Am 1976; 56: 1091-1130.

5. Gattinoni L, Mascheroni D, Torresin A, et al. Morphological response to positive end-expiratory pressure in acute respiratory failure: computerized tomography study. Intensive Care Med 1986; 12: 137-142.

6. Gattinoni L, Pesenti A, Torresin A, et al. Adult respiratory distress syndrome profiles by computed tomography. J Thorac Imag 1986; 1: 25-30.

7. Gattinoni L, Pesenti A, Bombino M, et al. Relationships between lung computed tomographic density, gas exchange and PEEP in acute respiratory failure. Anesthesiology 1988; 69: 824-832.

8. Gattinoni L, Pelosi P, Vitale G, Pesenti A, D'Andrea L, Mascheroni D. Body position changes redistribute lung computed tomographic density in patients with acute respiratory failure. Anesthesiology 1991; 74: 15-23.

9. Rinaldo JE, Rogers RM. Adult respiratory distress syndrome: changing concepts of lung injury and repair. $N$ Engl J Med 1982; 306: 900-909.

10. Rommelsheim K, Lakner K, Westhofen P, et al. Das respiratorische Distress-Syndrom des Erwachsenen (ARDS) im Computer-Tomogramth. Anaesth Intensivther Notfallmed 1983; 18: 59-644.

11. Maunder RJ, Shuman WP, McHugh JW, Marglin SI, Butler J. Preservation of normal lung region in adult respiratory distress syndrome: analysis by computed tomography. JAMA 1986; 255: 2463-2465.
12. Brismar B, Hedenstierna G, Lundquist H, et al. Pulmonary densities during anesthesia with muscular relaxation: a proposal of atelectasis. Anesthesiology 1985; 62: 422-428.

13. Strandberg A, Tokics L, Brismar B, et al. Constitutional factors promoting development of atelectasis during anesthesia and paralysis. Acta Anesthesiol Scand 1987; 31: 21-24.

14. Hedenstierna G. Gas exchange during anesthesia. Br J Anaesth 1990; 64: 507-514.

15. Gattinoni L, Pesenti A. ARDS: the dishomogeneous lung: facts and hypothesis. Intensive Crit Care Digest 1987; 6: $1-4$.

16. Gattinoni L, Pesenti A, Avalli L, et al. Pressure volume curve of total respiratory system in acute respiratory failure. Am Rev Respir Dis 1987; 36: 730-736.

17. Pelosi P, D'Andrea L, Vitale G, Pesenti A, Gattinoni L. Vertical gradient of regional lung inflation in adult respiratory distress syndrome. Am J Respir Crit Care Med 1994; 149: 8-13.

18. Jones R, Reid L, Zapol WM, Tomashefski JF, Kirton OC, Kobayashi K. Pulmonary vascular pathology: human and experimental studies. In: Zapol WM, Falke KJ, eds. Acute Respiratory Failure. New York, Basel, Marcel Dekker Inc., 1985; pp. 23-160.

19. Gattinoni L, Pelosi P, Pesenti A, et al. CT scan in ARDS: clinical and physiopathological insights. Acta Anaesth Scand 1991; 95: 87-96.

20. Jones T, Jones HA, Rhodes CG, Buckingham PD, Hughes JMB. Distribution of extravascular fluid volumes in isolated perfused lungs measured with $\mathrm{H}_{2} \mathrm{O}$. J Clin Invest 1976; 57: 706-713.

21. Hales CA, Devid JK, Ahlualia B, et al. Regional edema formation in isolated perfused dog lungs. Circ Res 1981; 48: 121-127.

22. Wollmer P, Rhodes CG, Deanfield J, et al. Regional extravascular density of the lung in patients with acute pulmonary edema. J Appl Physiol 1987; 63: 1890-1895.

23. D'Angelo E, Bonanni MV, Michelini S, Agostoni E. Topography of the pleural surface pressure in rabbits and dogs. Respir Physiol 1970; 8: 204-229.

24. Agostoni E. Mechanics of the pleural space. In: Macklem PT, Mead J, eds. Handbook of Physiology: The Respiratory System. Bethesda, Maryland, American Physiological Society, 1986; Section 3 (III): pp. 531-559.

25. Langer M, Mascheroni D, Marcolin R, Gattinoni L. The prone position in ARDS patients: a clinical study. Chest 1988; 94: 103-107.

26. Gattinoni L, Pelosi P, Valenza F, Mascheroni D. Patient Positioning in acute respiratory failure. In: Tobin MJ, ed. Principles and Practice of Mechanical Ventilation. New York,: McGraw Hill Inc., 1994; pp. 1067-1076.

27. Pappert D, Rossaint R, Slama K, Gruning T, Falke K. Influence of positioning on ventilation-perfusion relationships in severe adult respiratory distress syndrome. Chest 1994; 106: 1511-1516.

28. Rossi A, Ranieri VM. Positive end-expiratory pressure. In: Tobin MJ, ed. Principles and Practice of Mechanical Ventilation. New York, McGraw Hill Inc., 1994; pp. 259-303.

29. Ranieri VM, Eissa NT, Corbeil C, et al. Effects of PEEP on alveolar recruitment and gas-exchange in ARDS patients. Am Rev Respir Dis 1991; 144: 544-551.

30. Gattinoni L, Pesenti A, Baglioni S, Vitale G, Rivolta M, Pelosi P. Inflammatory pulmonary edema and positive end-expiratory pressure: correlations between imaging and physiologic studies. J Thorac Imag 1988; 3: 59-64.

31. Gattinoni L, D'Andrea L, Pelosi P, et al. Regional effects 
and mechanism of positive end-expiratory pressure in early adult respiratory distress syndrome. JAMA 1993; 269: 2122-2127.

32. Gattinoni L, Pelosi P, Crotti S, Valenza F. Effects of PEEP on regional distribution of tidal volume and recruitment in patients with adult respiratory distress syndrome. Am J Respir Crit Care Med 1995; 151: 1807-1814.

33. Pelosi P, Cereda M, Foti G, Giacomini L, Pesenti A. Alterations of lung and chest wall mechanics in patients with acute lung injury: effects of positive end-expiratory pressure. Am J Respir Crit Care Med 1995; 152: 532-537.
34. Broseghini C, Brandolese R, Poggi R, et al. Respiratory mechanics during the first day of mechanical ventilation in patients with pulmonary edema and chronic airway obstruction. Am Rev Respir Dis 1988; 138: 355-361.

35. Eissa NT, Ranieri VM, Corbeil C, et al. Analysis of the behavior of the respiratory system in ARDS patients: effects of flow, volume and time. J Appl Physiol 1991; 70: 2719-2729.

36. Gattinoni L, Bombino M, Pelosi P, et al. Lung structure and function in different stages of severe adult respiratory distress syndrome. JAMA 1994; 271: 1772-1779. 